\title{
Zur Kenntnis der Goldsulfide.
}

Von A. Gutbier und E. DÜrRwächter.

Der Verlauf der Einwirkung von Schwefelwasserstoff auf Lösungen von $\mathrm{Gold}(3)$-Chlorid, d. h. von $\mathrm{H}\left[\mathrm{AuCl}_{3} \cdot \mathrm{OH}\right]$, oder von Tetrachloroaurisäure, $\mathrm{H}\left[\mathrm{AuCl}_{4}\right]$, ist noch nicht sichergestellt: Man tindet Angaben, nach denen die Reaktion zum Metall ${ }^{1}$ ), zu Produkten mit wechselndem Schwefelgehalt ${ }^{2}$ ) und zu den Sulfiden $\mathrm{Au}_{2} \mathrm{~S}^{3}$ ) und $\left.\mathrm{Au}_{2} \mathrm{~S}_{2}{ }^{4}\right)$ führen soll. Das Sulfid $\mathrm{Au}_{2} \mathrm{~S}_{3}$ dagegen wurde auf diesem Wege bisher nicht gewonnen. Es ist ron Antony und Lucchesi aus dem Produkte der Behandlung von festem Lithiumtetrachloroauriat, $\mathrm{Li}\left[\mathrm{AuCl}_{4}\right]$, mit Schwefelwasserstoff und von K. A. HormanN und F. HöchtLEN ${ }^{6}$ ) bei der Umsetzung zwischen trockenem, in $\ddot{A}$ ther gelöstem Schwefelwasserstoff und ätherischen Lösungen von Tetrachloroaurisäure isoliert worden.

Nach Berzfiurus entsteht bei der Einwirkung von Schwefelwasserstoff auf wäBrige Lösungen von Gold(3)-Chlorid bei Siedehitze das Sulfid $\mathrm{Au}_{2} \mathrm{~S}$, nach Levor dagegen nach:

$$
8 \mathrm{AuCl}_{3}+3 \mathrm{H}_{2} \mathrm{~S}+12 \mathrm{H}_{2} \mathrm{O} \rightarrow 8 \mathrm{Au}+3 \mathrm{H}_{2} \mathrm{SO}_{4}+24 \mathrm{HCl}
$$

Metall. Letzterer Reaktionsverlauf wurde von HoFrMann und KRÜss für siedende Lösungen von Gold(3).Chlorid und langsam zutropfendes Schwefelwasserstoffwasser bestätigt.

OBERKaMPF andererseits gewann Präparate, die der Zusammensetzung $\mathrm{Au}_{2} \mathrm{~S}_{2}$ entsprachen, und HOFFMANN und KRÜss beobachteten die Bildung -des gleichen Sulfids, als sie Schwefelwasserstoff bei ge-

1) Levol, Ann. Chim. Phys. [3] 30 (1850), 356; L. Hoffmand und G. KRÜso, Ber. 20 (1887), 2361, 2704.

2) Feliengerg, Pogg. Ann. 50 (1840), 71; A. v. Schrötter und E. Pirfoznik, Ber. Wien. Akad. 1874, 165; Hofrmann und Krüss, 1. c.

3) Berzelius, Gmelin-Kraut 5, II, 269.

4) Oberkampe, Ann. Chim. Phys. 80 (1811), 140; Hoffmany und KrÜss, l. c.; A. Ditte, Compt. rend. 120 (1895), 320; U. Antony und A. Lucohesi, Gazz. chim. ital. 20 (1890), 61; 21 II (1891), 209.

5) Ber. 37 (1904), 245. 
wöhnlicher Temperatur auf Lösungen von Gold(3)-Chlorid einwirken ließen. Sie schlossen deshalb auf folgenden, durch Schwefelsäurebestimmungen wahrscheinlich gemachten Reaktionsverlauf:

$$
8 \mathrm{AuCl}_{3}+9 \mathrm{H}_{2} \mathrm{~S}+4 \mathrm{H}_{2} \mathrm{O} \rightarrow 4 \mathrm{Au}_{3} \mathrm{~S}_{2}+\mathrm{H}_{2} \mathrm{SO}_{4}+24 \mathrm{HCl} \text {. }
$$

Aber schon JACQUeLaIN ${ }^{l}$ ) hatte gefunden, und ANToNy und Lucchesi, sowie E. A. SchNeIDER ${ }^{2}$ ) bestätigten dies, daß die Goldsulfide bei unvollständiger Fällung mit noch vorhandenen GoldChlorverbindungen in der Wärme unter Bildung ron Metall und ron Schwefelsäure zu reagieren vermögen. ANTONY und LucchesI stellten für die Umsetzung die Gleichung:

$$
\mathrm{Au}_{2} \mathrm{~S}_{2}+4 \mathrm{AuCl}_{3}+8 \mathrm{H}_{2} \mathrm{O} \rightarrow 6 \mathrm{Au}+2 \mathrm{H}_{2} \mathrm{SO}_{4}+12 \mathrm{HCl}
$$

auf, durch die sie die Ergebnisse von Levor mittelbar bestätigten.

Bei unseren eigenen Untersuchungen haben wir die Bildung der Sulfide $\mathrm{Au}_{2} \mathrm{~S}$ und $\mathrm{Au}_{2} \mathrm{~S}_{2}$ nicht feststellen können. Wir fanden vielmehr folgendes:

1. Auf $2 \%$ ige Lösungen ron $\mathrm{H}\left[\mathrm{AuCl}_{4}\right], 4 \mathrm{H}_{2} \mathrm{O}$ in Normalsalzsäure wirkt ein lebhafter Schwefelwasserstoffstrom bei Zim. mertemperatur unter Abscheidung von schwarzen Niederschlägen ein, die immer metallglänzende Flitterchen entbalten und sich den Analysen nach als $\mathrm{Gemenge}$ von viel $\mathrm{Au}_{2} \mathrm{~S}_{3}$ mit wenig metallischem Gold erweisen. Eine geringe Menge von Schwefelsäure wird bei dieser Reaktion immer gebildet, aber weniger als der Gleichung (2) entspricht. Unter den angegebenen Bedingungen vollzieht sich also vorwiegend die Umsetzung:

$$
2 \mathrm{H}\left[\mathrm{AuCl}_{4}\right]+3 \mathrm{H}_{2} \mathrm{~S} \rightarrow \mathrm{Au}_{2} \mathrm{~S}_{3}+8 \mathrm{HCl},
$$

und der immer festgestellte Gehalt der Fällungsprodukte an freiem Metall und der der Filtrate an Schwefelsäure ist, wie sich noch zeigen wird, auf die sekundäre Reaktion zwischen dem Sultid und noch unveränderter Chlorosäure zurückzuführen.

Ein langsamer Schwefelwasserstofistrom ruft in den gleichen Lösungen bei Siedehitze zunächst und vorübergehend ebenfalls Bildung des Sulfids hervor, das sich aber sogleich mit der im Überschusse vorhandenen Chlorosäure weiter umsetzt, so daB schlieBlich reines Metall vorliegt und das Filtrat entsprechend bedeutende Mengen von Schwefelsäure enthält.

1) Compt. rend. 14 (1842), 642.

2) Ber. 24 (1891), 2241. 
Wird ein etwas lebhafterer Schwefelwasserstoffstrom in Lösungen derselben Zusammensetzung eingeleitet, somit eine schnellere Abnahme der Konzentration der Flüssigkeit an $\mathrm{H}\left[\mathrm{AuCl}_{4}\right]$ ermöglicht als im vorhergehenden Falle, so ist in dem Reaktionsprodukt auch bei erhöhter Temperatur immer das schwarze Sulfid $\mathrm{Au}_{2} \mathrm{~S}_{2}$ enthalten. Man gewinnt unter diesen Umständen Gemische von Sulfid und Metall, deren Zusammensetzung von der Temperatur, der Konzentration der Ausgangslösung an $\mathrm{H}\left[\mathrm{AuCl}_{4}\right]$ und der Geschwindigkeit des Gasstromes abhängig ist. Alle diese Präparate lassen den Gehalt an freiem Metall und an Sulfid jederzeit bei der mikroskopischen Prüfung einwandfrei erkennen.

Temperaturerniedrigung auf $-2^{\circ}$ bis $-4^{\circ}$ und möglichst schnelle Verminderung der Konzentration der Lösungen an $\mathrm{H}\left[\mathrm{AuCl}_{4}\right]$ durch einen sehr lebhaften Schwefelwasserstoffstrom ermöglichen die Bereitung recht reiner Präparate des Sulfids $\mathrm{Au}_{\mathbf{z}} \mathrm{S}_{\mathbf{z}}$.

2. Auf $2 \%$ ige wäBrige Lösungen von Gold(3)-Chlorid wirkt Schwefelwasserstoff, auch bei $-2^{\circ}$ und in lebhaftem Strome eingeleitet, immer unter Bildung von metallhaltigem Sulfid $\mathrm{Au}_{2} \mathrm{~S}_{3}$ ein. Die Möglichkeit, unter diesen Versuchsbedingungen die sekundäre Reaktion auszuschalten und zum reinen Sulfid zu gelangen, bestand aus den unter 3. angeführten Gründen nicht. Und ebensowenig vermochten wir die Ergebnisse von HoFFmann und KRîss') zu reproduzieren, d. h. die Bildung des Sulfids $\mathrm{Au}_{2} \mathrm{~S}_{3}$ nachzuweisen.

3. Besonders wichtig erschien die quantitative Verfolgung der sekundären Reaktion, d.h. der Umsetzung des Sulfids mit dem GoldChlorverbindungen: Die ron ANTONY und LUCCHESI aufgestellte Gleichung (3) muBte, da das Reaktionsprodukt unseren Befunden nach nicht $\mathrm{Au}_{2} \mathrm{~S}_{2}$, sondern $\mathrm{Au}_{2} \mathrm{~S}_{3}$ darstellt, umgeformt werden in:

$\mathrm{Au}_{2} \mathrm{~S}_{8}+6 \mathrm{H}\left[\mathrm{AuCl}_{3} \cdot \mathrm{OH}\right]+6 \mathrm{H}_{8} \mathrm{O} \rightarrow 8 \mathrm{Au}+3 \mathrm{H}_{8} \mathrm{SO}_{4}+18 \mathrm{HCl}$ bzw.

$$
\mathrm{Au}_{2} \mathrm{~S}_{3}+6 \mathrm{H}\left[\mathrm{AuCl}_{4}\right]+12 \mathrm{H}_{2} \mathrm{O} \rightarrow 8 \mathrm{Au}+3 \mathrm{H}_{2} \mathrm{SO}_{4}+24 \mathrm{HCl},
$$

doch entspricht der Reaktionsverlauf unseren Versuchsergebnissen nach nicht diesen Gleichungen: Die bei der Temperatur des siedenden Wasserbads gefundenen Goldmengen blieben hinter den berechneten immer weit zurïck. Sicherlich spielt sich die Umsetzung sehr viel verwickelter $a b$, als eine einfache chemische Gleichung zusammenzufassen gestattet. Experimentell aber lieb sich nur fest-

1) Vielleicht hat die angewandte analytische Methode den genannten Forschern einen zu hohen Schwefelsäuregehalt im Filtrate vorgetäuscht. 
stellen, daB die Reaktion in ihren Endergebnissen einen Ausdruck in den Gleichungen:

$4 \mathrm{Au}_{2} \mathrm{~S}_{3}+22 \mathrm{H}\left[\mathrm{AuCl}_{3} \cdot \mathrm{OH}\right]+23 \mathrm{H}_{2} \mathrm{O}+3 \mathrm{O} \rightarrow 30 \mathrm{Au}+12 \mathrm{H}_{2} \mathrm{SO}_{4}+66 \mathrm{HCl}$ bzw.

$4 \mathrm{An}_{2} \mathrm{~S}_{3}+22 \mathrm{H}\left[\mathrm{AuCl}_{4}\right] \quad+45 \mathrm{H}_{2} \mathrm{O}+3 \mathrm{O} \rightarrow 30 \mathrm{Au}+12 \mathrm{H}_{2} \mathrm{SO}_{4}+88 \mathrm{HCl}$

finden dürfte.

Weiter stellten wir fest, daB wäßrige Lösungen ron Gold(3)Chlorid auf das Sulfid schneller einwirken als gleichkonzentrierte Lösungen der Tetrachloroaurisäure in Normalsalzsäure bei derselben Temperatur. In dieser Tatsache finden die unter 2. beschriebenen Erscheinungen ihre Erklärung.

Bei der unmittelbaren Einwirkung von Schwefelwasserstoff auf die Lösungen der Gold-Chlorverbindungen wird die sekundäre Reaktion beschleunigt durch Steigerung der Temperatur, Erhöhung der Konzentration der Ausgangslösung und Verminderung der Geschwindigkeit des Gasstroms. Durch geeignete Einstellung aller in Betracht kommenden Komponenten läBt sich die Reaktion so leiten, daß das Endprodukt entweder reines schwefelfreies Metall oder Gemenge von Gold mit dem Sulfid $\mathrm{Au}_{2} \mathrm{~S}_{3}$ in beliebigen Ver$\mathrm{hältnissen} \mathrm{oder} \mathrm{das} \mathrm{Sulfid} \mathrm{Au}_{2} \mathrm{~S}_{3}$ darstellt.

Mit dieser Erkenntnis sind u. E. die groben Unterschiede in den Ergebnissen älterer Arbeiten aufgeklärt.

\section{Experimentelles.}

(Bearbeitet ron E. DÜRnWÄchter.)

I. Einwirkung von Schwefelwasserstoff auf $2 \%$ ige Lösungen von Tetrachloroaurisäure, $\mathrm{H}\left[\mathrm{AuCl}_{4}\right], 4 \mathrm{H}_{2} \mathrm{O}$ in Normalsalzsäure.

a) Ein lebhafter Schwefelwasserstoffistrom') bewirkt bei gewöhnlicher Temperatur zunächst Bildung eines dünnen braunen, in Regenbogenfarben schillernden Häutchens an der Oberfäche der Lösung. Die nächsten Gasblasen rufen eine braune Trübung hervor, und bald scheidet sich ein brauner Niederschlag ab, der schnell ruBartig tiefschwarz wird. Man digeriert die Fällung mit Wasser, wäscht sie säurefrei, zieht sie nach der Behandlung mit Alkohol und Äther im Soxlethapparat mit Schwefelkohlenstoff aus und trocknet sie nach erneutem Abwaschen mit Äther in der Luftleere über Phosphorpentoxyd. bereitet.

1) Das Gas wurde aus reinem Natriumsulfid und arsenfreier Salzaäure 
So vorbereitete Präparate bestehen aus schwarzen amorphen Blättchen und wenigen metallglänzenden Flitterchen. Sie sind bei $90^{\circ}$ noch beständig, zeigen bei $110^{\circ}$ Spuren von Zersetzung, werden zwischen $170^{\circ}$ und $200^{\circ}$ tiefbraun und sind bei $250^{\circ}$ schon ziemlich vollständig zersetzt.

$0,7136 \mathrm{~g}$ der exsikkatortrockenen Substanz erlitten bei sechsstündigem Erwärmen auf $90^{\circ}$ einen Gewichtsverlust von $0,0010 \mathrm{~g}=0,14^{\%}$. Sie wogen nach je sechsstündigem Erhitzen auf:

$\begin{array}{ccccccc}110 & 130 & 150 & 170 & 200 & 230 & 250^{\circ} \\ 0,7079 & 0,7008 & 0,6012 & 0,5902 & 0,5863 & 0,5854 & 0,5745 \mathrm{~g} .\end{array}$

Die Analysen konnten nach dem von HofFmass und KRÜss vorgeschlagenen Verfahren nicht ausgeführt werden, da das Sulfid mit rauchender Salpetersäure unter Feuererscheinung und mit der konzentrierten Säure auch noch sehr lebhaft reagiert. Nach mancherlei anderen, von MiBerfolgen begleiteten Versuchen gelangen die Bestimmungen schließlich dadurch, daß man die in Porzellanschiffchen eingewogene, fein gepulverte Substanz in Röhren aus schwer schmelzbarem Glase sehr langsam und äuBerst vorsichtig in reinem Sauerstoff erhitzte und das gebildete Schwefeldioxyd in einer vorgelegten LUNGEschen Zehnkugelröhre mit Wasserstoffperoxyd oxydierte. Im Schiffchen blieb bei richtig geleiteter Operation reines Metall zurück.

$0,2921 \mathrm{~g}$ Sbst. enthielten $0,2360 \mathrm{~g} \mathrm{Au}$ und $0,0557 \mathrm{~g} \mathrm{~S} .-0,2719 \mathrm{~g}$ Sbst. enthielten $0,2208 \mathrm{~g}$ Au und $0,0491 \mathrm{~g}$ S. $-0,2197 \mathrm{~g}$ Sbst. enthielten $0,1773 \mathrm{~g}$ Au und 0,0416 g S. - 0,3205 g Sbst. enthielten 0,2615 g Au und 0,0582 g S. $0,6346 \mathrm{~g}$ Sbst. enthielten $0,5065 \mathrm{~g} \mathrm{Au}$ und $0,1213 \mathrm{~g} \mathrm{~S}$.

Berechnet für $\mathrm{Au}_{8} \mathrm{~S}_{8}$ : Au 80,39\%. Gefunden: 80,79, 81,20, 80,70,81,59, 79,81\%. $\mathrm{S} 19,61 \%$. $\quad 19,07,18,06,1 \times, 93,18,16,19,11 \%$.

Die wasserklaren Filtrate der einzelnen Sulfidfällungen wurden entsprechend eingeengt und zu Schwefelsäurebestimmungen benutzt. Die gewogenen Mengen von Bariumsulfat betrugen in allen Fällen viel weniger, als Gleichung (2) verlangen würde.

b) Beim Einleiten eines langsamen Gasstromes bei Siedehitze besteht die Fällung aus reinem metallischen Gold.

c) Mit Steigerung der Geschwindigkeit des Schwefelwasserstoffstroms wird auch bei erhöhter Temperatur ein schwefelhaltiger Niederschlag gewonnen. Er stellt Gemische des Sulfids $\mathrm{Au}_{2} \mathrm{~S}_{3}$ mit Gold dar, und sein Sulfidgehalt schwankt mit der Temperatur, mit der Geschwindigkeit des Gasstromes und, wie 
gesondert festgestellt wurde, mit der Konzentration der Ausgangslösung. Bedingungen, die zu einheitlichen Sulfiden $\left(\mathrm{Au}_{2} \mathrm{~S}\right.$ oder $\mathrm{Au}_{2} \mathrm{~S}_{2}$ ) führen würden, ließen sich nicht ausfindig machen. In allen Präparaten konnte die Gegenwart von metallischem Gold bei der mikroskopischen Untersuchung einwandfrei festgestellt werden.

d) Leitet man einen schnellen Schwefelwasserstoffstrom bei $-2^{\circ}$ bis $-4^{0}$ in Normalsalzsäure ein, und läBt man gleichzeitig die ebenfalls gekühlte Lösung der Chlorosäure langsam zufließen, so kann die zur Bildung ron Metall führende Nebenreaktion weitgehend ausgeschaltet werden. Unter der nötigen Vorsicht auf solche Weise bereitete, wie oben beschrieben gereinigte Präparate sind amorph, gleichmäBig tiefschwarz und lassen unter dem Mikroskop keinerlei fremde Beimengungen erkennen.

$0,1553 \mathrm{~g}$ Sbst. enthielten $0,1246 \mathrm{~g}$ Au und $0,0302 \mathrm{~g} \mathrm{~S}$. - 0,1404 g Sbst. enthielten $0,1127 \mathrm{~g}$ Au und $0,0276 \mathrm{~g} \mathrm{~S}$. - 0,1602 $\mathrm{g}$ Sbst. enthielten 0,1286 $\mathrm{g}$ $\mathrm{Au}$ und $0,0313 \mathrm{~g} \mathrm{~S}$.

Berechnet für $\mathrm{Au}_{\mathrm{z}} \mathrm{S}_{\mathrm{s}}$ : $\begin{gathered}\mathrm{Au} \\ \mathrm{S} 19,39 \%\end{gathered}$

Das Sulfid wird von Salzsäure, Schwefelsäure und verdünnter Salpetersäure bei Zimmertemperatur nicht angegriffen. Konzentrierte Salpetersäure zersetzt es unter lebhafter Entwicklung von Stickoxyden zu Gold und Schwefelsäure. Rauchende Salpetersäure wirkt unter prächtiger Feuererscheinung ein, und nach beendeter Reaktion findet man zuweilen schön ausgebildete Goldkriställchen vor. Durch Königswasser wird das Sulfid in Tetrachloroaurisäure und Schwefelsäure in der Weise verwandelt, dab das erstere Produkt ziemlich schnell gebildet wird, während die Hauptmenge des Schwefels nur langsam verschwindet. In entsprechender Weise wirken Bromsalzsäure und Bromwasser.

Konzentrierte Lösungen von Natriumsulfid lösen die Niederschläge bei gewöhnlicher Temperatur langsam, in der Wärme schneller zu braunen Flüssigkeiten auf, die bei längerem Erhitzen gelbgrün werden; die verdünnten Lösungen sind farblos. Verdünnte Salzsäure scheidet aus Flüssigkeiten dieser Art feinst verteilten Schwefel und hellbraune flockige Niederschläge aus. Von Alkalipolysulfidlösungen wird das Sulfid bei Zimmertemperatur langsam, etwas leichter in der Wärme aufgenommen. Beim Erhitzen mit konzentrierten Alkalilaugen entstehen schwachgelbe Flüssigkeiten, und schwefelfreies Gold bleibt zurück.

Konzeutrierte und auch verdünnte Kaliumcyanidlösungen nehmen 
das Sulfid schnell und vollständig auf. Die Flüssigkeiten färben sich hierbei zunächst tief dunkelbraun, hellen jedoch nach einiger Zeit, schneller beim Erwärmen, wesentlich auf und werden weingelb. In ihnen konnte der Nachweis des Rhodanions positiv gefuhrt werden, und eine größere Menge des Sulfids in der eben ausreichenden Menge von Kaliumcyanid, in der Wärme gelöst, schied beim Erkalten die bekannten Kristalle von $\mathrm{K}\left[\mathrm{Au}(\mathrm{CN})_{2}\right]$ ab. Während Schwofel- und Salpetersäure aus den Lösungen nur feinen Schwefel abscheiden, gestaltet sich die Reaktion mit Salzsäure verwickelter. Wir haben sie näher untersucht und folgendes festgestellt. Fügt man zu einer mäBig verdünnten Lösung des Sulfids in Kaliumcyanid bei Siedehitze schnell überschüssige konzentrierte Salzsäure hinzu, so wird die Flüssigkeit schnell gelb, beginnt zu opalisieren und scheidet nach einiger Zeit einen braunen amorphen Niederschlag $a b$, der, leicht löslich in Kaliumcyanid, ein Gemenge ron Gold, Gold(1)-Cyanid und Schwefel darstellt. LäBt man andererseits zu der mäßig verdünnten Lösung überschüssige $20 \%$ ige Salzsäure hinzulaufen, so scheidet die nun gelbe, opalisierende Flüssigkeit bei schwachem Erwärmen langsam metallisches Gold in kleinen Kriställchen ab. Wirkt schlieBlich auf die verdünnten Lösungen bei Zimmertemperatur verdünntere Salzsäure, so färbt sich die Flüssigkeit zunächst reingelb und scheidet dann in dem Mabe, in dem Cyanwasserstoff entweicht, Gold(1)-Cyanid aus.

\section{Einwirkung von Schwefelwasserstoff auf $2 \%$ ige wäBrige Lösungen von Gold(3)-Chlorid.}

In $2 \%$ igen wäBrigen Lösungen des reinen, nach J. Thomsen ${ }^{1}$ ) bereiteten Chlorids ruft ein lebhafter Strom von Schwefelwasserstoff bei $-2^{0}$ die Bildung von amorphen schwarzen Niederschlägen hervor, die wie die übrigen Präparate zur Analyse vorbereitet, bei der Betrachtung unter dem Mikroskope einen geringen Gehalt an kleinen metallglänzenden Flittern immer erkennen lassen. Die sonstigen Eigenschaften der hier isolierten Produkte entsprechen denen der früher beschriebenen.

$0,1917 \mathrm{~g}$ Sbst. enthielten $0,1550 \mathrm{~g} \mathrm{Au}$ and $0,0351 \mathrm{~S}$. $-0,2042 \mathrm{~g}$ Sbst. enthielten 0,165j $\mathrm{g}$ Au und $0,0378 \mathrm{~g} \mathrm{~S}$. - 0,1995 $\mathrm{g}$ Sbst. enthielten 0,1619 g Au und $0,0356 \mathrm{~g} \mathrm{~S}$. $-0,1785 \mathrm{~g}$ Sbst. enthielten $0,1455 \mathrm{~g}$ Au und $0,0328 \mathrm{~g} \mathrm{~S}$.

Berechnet für $\mathrm{Au}_{2} \mathrm{~S}_{8}$ : Au 80,39\%. Gefunden: 80 86, 81,07, 81,15, 81,51\%. S $19,61 \%$. $\quad 18,31,18,51,17,84,18,38 \%$.

1) J. prakt. Chem. [2] 13 (1876), 337. 
Wieder wurden auch hier die wasserhellen Filtrate der Sulfidlösungen auf ihren Schwefelsäuregehalt untersucht, und abermals wurde festgestellt, daB dieser mit dem nach Gleichung (2) berechneten nicht in Übereinstimmung zu bringen war.

Es gelang unter keinen Bedingungen, die Verunreinigang so entstehender Präparate mit Metall vollkommen auszuschlieBen. Mit Erhöhung der T'emperatur und Konzentration der Ausgangslösungen bei gleichbleibender Stärke des Gasstroms, wie bei gleichgehaltener Temperatur und Konzentration unter Verminderung der Geschwindigkeit des Schwefelwasserstoffstroms, steigt der Gehalt der Niederschläge an freiem Metall uud damit auch die Menge der Schwefelsäure im Filtrate an. Bei Siedehitze und langsamem Schwefelwasserstoffstrome stellt das aus $2 \%$ igen Lösungen gefällte Produkt reines schwefelfreies Metall dar.

Bedingungen, die zu einheitlichen Sulfiden von der Zusammensetzung $\mathrm{Au}_{2} \mathrm{~S}$ oder $\mathrm{Au}_{2} \mathrm{~S}_{2}$ führen würden, ließen sich auch hier nicht auffinden.

\section{Umsetzung des Sulflds mit Lösungen von Gold(3)-Chlorid oder Tetrachloroaurisäure.}

Alle unsere Versuche ergaben, daB bei gleicher Temperatur und Konzentration Lösungen von Gold(3)-Chlorid schneller als solche von Tetrachloroaurisäure auf das Sulfid einwirken. Diese Tatsache tritt in besonders auffälligem Maße bei Temperaturen von $0^{\circ}$ und darunter in Erscheinung. Bei Zimmertemperatur schreitet die Um setzung nur langsam fort, läBt sich auch durch Rühren der Gemischenicht auffällig beschleunigen und führt deshalb auch nicht zu reinem schwefelfreien Metall. Bei Siedehitze dagegen überzieht sich das in Lösungen der Gold-Chlorverbindungen eingebrachte Sulfid augenblicklich mit einem Hauche von metallischem Gold und ist, je nach der Konzentration der Flüssigkeit, in längerer oder kürzerer Zeit vollständig in Metall verwandelt.

Zur quantitativen Verfolgung der Zusammensetzung wurden bestimmte Mengen des nach I. d) dargestellten Sulfids mit wenig mehr als der nach Gleichung (6) berechneten Menge $\mathrm{H}\left[\mathrm{AuCl}_{4}\right], 4 \mathrm{H}_{2} \mathrm{O}$ in $30 \mathrm{ccm}$ Normalsalzsäure auf dem Wasserbade 14 bis 16 Stunden lang erhitzt. Nach dieser Zeit war das Sulfid bei den weiter unten mitgeteilten Bestimmungen quantitativ in Metall verwandelt, wovon man sich durch Kontrollbestimmungen überzeugte. Im Filtrat konnte Schwefelsäure unmittelbar gefällt werden, vorausgesetzt, daB nicht 
274 A. Gutbier und E. Dürrwächter. Zur Kenntnis der Goldsulfide.

zuviel Tetrachloroaurisäure unverändert geblieben war, die sonst mit Hydrazinhydrat reduziert werden muBte. Alle gewogenen Niederschläge von Bariumsulfat waren rein weib.

Wir stellen in der folgenden Tabelle einige in dieser Weise durchgeführte Bestimmungen zusammen. Ihre Ergebnisse sprechen mit Wahrscheinlichkeit für Gleichung (8).

\begin{tabular}{|c|c|c|c|c|c|c|}
\hline \multirow{2}{*}{$\begin{array}{l}\text { Angewand } t \\
\text { g } \mathrm{Au}_{2} \mathrm{~S}_{3}\end{array}$} & \multirow{2}{*}{$\begin{array}{l}\text { ÜberschuB an } \mathrm{g} \\
\mathrm{H}[\mathrm{AuCl}], 4 \mathrm{H}_{2} \mathrm{O}\end{array}$} & \multicolumn{2}{|c|}{ Gefunden $g$} & \multicolumn{3}{|c|}{ Berechnet $\mathrm{g}$} \\
\hline & & $A \mathbf{n}$ & $\mathrm{H}_{2} \mathrm{SO}_{4}$ & $\underset{\operatorname{mach}(6)}{A \mathfrak{u}}$ & $\underset{\text { nach (8) }}{\mathrm{Au}}$ & $\mathrm{H}_{2} \mathrm{SO}_{4}$ \\
\hline 0,2 & 0,01 & 0. & - & 0, & 0,7723 & - \\
\hline & & & 0,1 & & 0 & 0,1 \\
\hline 0,22 & 0, & 0,6 & 0,1346 & 0,7287 & 0,6831 & 0,1359 \\
\hline 0,2570 & 0,24 & 0,7636 & 0,1539 & 0,8265 & 0,7748 & 0,1541 \\
\hline 0,2002 & 0,07 & 0,6006 & 0,1 & 0,64 & 0,6035 & 0,1201 \\
\hline 0,2006 & 0,07 & 0,6070 & 0,1196 & 0,6451 & 0,6048 & 0,1203 \\
\hline
\end{tabular}

Stuttgart, Laboratorium für anorganische Chemie der Iechnischen Hochschule.

Bei der Redaktion eingegangen am 17. März 1922. 